\title{
Análise da alteração postural na coluna lombar das gestantes
}

\section{Analysis of postural changes on lumbar spine of pregnant women}

\section{Análisis de la alteración postural en la columna lumbar de las embarazadas}

Recebido: 28/07/2018

Aprovado: 09/02/2018 Publicado: 13/05/2019

Max dos Santos Afonso ${ }^{1}$ Caroline Henriques Graciano ${ }^{2}$ Karine Fernandes Ávila ${ }^{3}$ Marla dos Santos Afonso ${ }^{4}$ Abelardo Soares de Oliveira Júnior ${ }^{5}$ Fernanda Burlani Neves 6

0 presente trabalho teve como objetivo analisar a alteração postural na coluna lombar de gestantes por meio da biofotogrametria nos três trimestres gestacionais e verificar a frequência de lombalgia. Trata-se de um estudo quantitativo composto por 21 gestantes, realizado entre setembro a novembro de 2012, através dos questionários sobre o perfil da população, nórdico de sintomas osteomusculares e o internacional de atividade física. Foi feita palpação e localização dos pontos anatômicos para colocação de marcadores, após as gestantes foram submetidas ao registro fotográfico e os ângulos analisados através do Software de Avaliação Postural. Foi encontrado alto índice de dor na região lombar das gestantes, em um total de $72 \%$, sendo que todas apresentaram aumento na lordose lombar. Foram verificadas alterações posturais significativas entre os três trimestres gestacionais e a presença de algia lombar foi referida pela maioria das gestantes.

Descritores: Gestantes; Dor lombar; Exercício; Fotogrametria.

This study aimed to analyze the postural change in lumbar spine of pregnant women through photogrammetry in the three trimesters of pregnancy and check the frequency of low back pain. This is a quantitative study, consisting of 21 pregnant women, carried out from September to November 2012, using questionnaires on the profile of the population, the Nordic Musculoskeletal Questionnaire and the International Physical Activity Questionnaire. Palpation and location of anatomical points were made for placing markers. After that, pregnant women were subjected to the photographic record and the angles analyzed using the Postural Assessment Software. A high index of pain in the lumbar region of pregnant women was found for a total of $72 \%$, with all showing an increase in lumbar lordosis. Significant postural changes among the three trimesters of pregnancy were verified and the presence of low back pain was reported by the majority of pregnant women.

Descriptors: Pregnant women; Low back pain; Exercise; Photogrammetry.

El presente trabajo lleva como objetivo analizar la alteración postural en la columna lumbar de gestantes a través de la biofotogrametría en los tres bimestres gestacionales y verificar la frecuencia de lumbalgia. Se trata de un estudio cuantitativo compuesto por 21 gestantes, realizado entre septiembre hasta noviembre de 2012, a través de encuesta acerca del perfil de la población, nórdico de síntomas osteomusculares y el internacional de actividad física. Fue hecha palpación y ubicación de los puntos anatómicos para ponerse marcadores, tras eso, las embarazadas fueron sometidas al registro fotográfico y los ángulos analizados a través del Software de Evaluación Postural. Fue encontrado alto índice de dolor en la región lumbar de las gestantes, en un total del $72 \%$, siendo que todas presentaron aumento de lordosis lumbar. Fueron verificadas alteraciones posturales significativas entre los tres trimestres gestacionales y la presencia de algia lumbar fue referida por la mayoría de las embarazadas.

Descriptores: Mujeres embarazadas; Dolor de la región lumbar; Ejercicio; Fotogrametría

1. Fisioterapeuta. Especialista em Fisioterapia Desportiva. Mestrando em Enfermagem pela Universidade Federal do Rio Grande (FURG). Docente do Curso de Fisioterapia da Faculdade Anhanguera de Pelotas, Rio Grande do Sul, Brasil. ORCID: 0000-0001-9989-9535 E-mail: max.afonso@hotmail.com

2. Fisioterapeuta. ORCID: 0000-0002-5558-0968 E-mail: carolhgraciano@yahoo.com.br

3. Fisioterapeuta. Especialista em Fisioterapia Hospitalar. ORCID:0000-0002-7772-1434 E-mail: kaka.fa@hotmail.com

4. Psicóloga. Mestranda em Enfermagem pela FURG, Porto Alegre, RS, Brasil. ORCID:0000-0002-7014-5999 E-mail: marla_afonso@hotmail.com

5. Fisioterapeuta. Especialista em Saúde Pública. Especialista em Atenção ao Câncer Infantil. Mestrando em Ciências Pneumológicas pela Universidade Federal do Rio Grande do Sul, Porto Alegre, RS, Brasil. ORCID:0000-0003-4682-688X E-mail: junior_osoares@hotmail.com

6. Fisioterapeuta. Mestre em Ciências da Saúde. Doutora em Saúde e Comportamento. Docente do Curso de Fisioterapia da Faculdade Anhanguera de Pelotas, RS, Brasil. ORCID:0000-0003-0468-4676 E-mail: fernanda.burlani@aedu.com 


\section{INTRODUÇÃO}

A gestação é um período em que a mulher sofre diversas alterações que envolvem os variados sistemas e aparelhos. Essa mulher vive um período com transformações do aspecto biológico e psíquico e essas repercussões variam de uma gestante para outra e da idade gestacional ${ }^{1}$.

A mulher era desencorajada a prática de exercícios físicos durante o período gestacional, devendo recolher-se ao repouso sedentário, visto que, os esforços físicos eram vistos como prejudiciais ao feto ${ }^{2}$.

As mudanças fisiológicas são necessárias para adaptar a mulher a esta nova condição. Essas transformações envolvem adaptações hemodinâmicas, hormonais e biomecânicas, como por exemplo, erguer objetos, tossir, movimentos rápidos de tronco, produzem forças que desafiam o controle postural e que podem gerar desordens musculoesqueléticas, como a lombalgia ${ }^{3,4}$.

Existem três classificações para a lombalgia gestacional: a dor pélvica, a dor lombar e a combinação de ambas. A dor pélvica é inconstante e, pode ter a irradiação da dor para os glúteos e membros inferiores, causando bloqueio e interferindo na marcha e acometendo a postura estática. A lombalgia ou dor lombar estabelece distúrbios musculoesqueléticos localizados abaixo das últimas costelas e acima da prega glútea. Apresenta-se por meio da dor à palpação, rigidez articular, tensão muscular, diminuição da amplitude de movimento da coluna, podendo interferir na postura e na marcha 5 .

Biomecanicamente, uma das principais causas das mudanças na estática e na dinâmica do esqueleto da gestante, é o constante crescimento do útero. Ocorre um deslocamento do centro de gravidade para cima e para frente, devido ao aumento do abdome, ganho de peso, alterações hormonais e aumento das mamas, o que leva a alterações de postura. Esses ajustes geram uma acentuação da lordose lombar e consequente tensão da musculatura paravertebral ${ }^{6}$.
A partir do segundo trimestre gestacional, a sobrecarga nos músculos e ligamentos da coluna vertebral é mais acentuado pois o hormônio relaxina é secretado mais intensamente no início da gestação e acaba atingindo um equilíbrio no segundo trimestre permanecendo até o final do período gravídico ${ }^{2-7}$.

Sob a ação dos hormônios, principalmente à da relaxina existe um relaxamento crescente dos ligamentos, além de um amolecimento cartilaginoso e aumento no volume de líquido sinovial e no espaço articular, agindo sobre as fibras colágenas, diminuindo sua densidade e ocasionando maior extensibilidade das estruturas articulares. 0 resultado é uma mobilidade articular aumentada e articulações mais instáveis, predispondo as gestantes às lesões sendo que altos índices de relaxina podem estar associados à dor lombar e pélvica 2-7.

Ainda que essa condição dolorosa não esteja totalmente esclarecida sua etiologia está correlacionada a fatores fisiológicos, biomecânicos, vasculares e psicológicos. Estima-se que a lombalgia gestacional ataca uma a cada duas gestantes, em geral a partir do terceiro trimestre de gestação, causando preocupação e desconforto tanto por sua alta prevalência quanto pela intensidade causada pela dor. De acordo com pesquisas realizadas no Brasil, a prevalência encontrada sobre o aparecimento de lombalgia durante a gestação varia de $48 \%$ a $83 \%{ }^{8}$.

Tendo em vista as repercussões que tais sintomatologias podem gerar na gestante, justifica-se a busca de métodos e instrumentos para a avaliação e a identificação das alterações posturais advindas do período gestacional.

Devido à importância de se compreender melhor a relação entre as alterações posturais e os sintomas da lombalgia gestacional, o presente trabalho teve como objetivo analisar a alteração postural na coluna lombar de gestantes por meio de biofotogrametria nos três trimestres gestacionais e verificar a frequência da lombalgia. 


\section{MÉTODO}

Trata-se de um estudo transversal, quantitativo e descritivo realizado com gestantes em acompanhamento pré-natal, no período entre setembro a novembro de 2012, nas Unidades Básicas de Saúde (UBS) dos Bairros São Miguel, Castelo Branco II e Santa Rosa, localizados no município de Rio Grande, RS, Brasil.

A identificação das pacientes para composição da amostra foi realizada por meio de consulta nas fichas de registros de avaliação da unidade. As pacientes identificadas foram abordadas após seu atendimento na UBS.

Os critérios de inclusão no estudo foram: ter idade igual ou superior a 18 anos, estar vinculadas ao serviço de saúde, sem complicações gestacionais. Para maior confiabilidade, foi conferido no prontuário dos indivíduos participantes, evoluções médicas sobre o andamento do período gestacional.

Como instrumentos de pesquisa foram utilizados os seguintes instrumentos: Questionário sobre o Perfil da População, abordando questões socioeconômicas, demográficas, comportamentais e de saúde da gestante; Questionário Nórdico de Sintomas Osteomusculares (QNSO). Este compõe-se em escolhas múltiplas ou binárias quanto à incidência de sintomas regiões anatômicas do corpo nos doze últimos meses, bem como o afastamento de suas atividades normais ${ }^{12,13}$

E, utilizou-se ainda o Questionário Internacional de Atividade Física (IPAQ); para estimar o nível habitual de atividade física, medida em minutos por semana, considerando que a gestante que realizar durante a semana 150 minutos ou mais de atividade, é considerada ativa fisicamente ${ }^{14,15}$.

A Biofotogrametria Computadorizada foi utilizada para diagnóstico físico funcional, pois ela fundamenta-se na aplicação do princípio fotogramétrico às imagens fotográficas, obtidas de movimentos corporais, onde se realizam as bases apropriadas para a fotointerpretação. É usada na avaliação postural e biomecânica do corpo, pois fornece valores precisos que são fundamentais no direcionamento de um determinado tratamento.

Trata-se de um método de avaliação postural de alta complexidade e confiabilidade nos seus respectivos diagnósticos e está embasada em um algoritmo matemático que transforma pontos de imagens em eixos coordenados cartesianos e os quantifica angularmente. Referências ósseas, articulares, planos, eixos e regiões corporais podem ser demarcados nos indivíduos avaliados pela Fotogrametria Computadorizada, anteriormente à coleta de imagens ou ainda diretamente na imagem, após a coleta. Esta demarcação é fundamental para a operacionalização do estudo e análise dos dados e, depende do conhecimento da anatomia palpatória ${ }^{9-11}$.

Durante a coleta, as imagens foram digitalmente armazenadas para posterior análise por meio do Software para Análise Postural - (Posture Analysis Software SAPo/PAS), de acesso livre e gratuito, fabricado para exame de imagens. 0 software foi elaborado exclusivamente para análises de imagens digitais aplicadas à área da saúde e pode ser arquivado para acompanhamento da evolução postural; além disso, apresenta validação e bons níveis de reprodutibilidade ${ }^{16,17}$. Para o estudo da massa corporal atual e da estatura, foi utilizada uma balança antropométrica.

As participantes da pesquisa foram divididas em três grupos, de acordo com o trimestre gestacional, denominado (TG). Sendo o grupo do primeiro trimestre gestacional (1TG - 1 a 12 semanas de gestação); o grupo do segundo trimestre (2TG - 13 a 28 semanas de gestação); e o grupo do terceiro trimestre gestacional (3TG - da 29a semana até o parto).

A marcação dos pontos anatômicos foi feita com bolinhas de isopor com 1 centímetro de diâmetro, e para demarcação dos pontos na coluna vertebral total (torácicas T7, T12), (lombares L2, L3, L4 e L5), foi utilizada etiqueta na cor verde fluorescente da marca Pimaco.

0 estudo foi aprovado pela Secretaria de Saúde do Município de Rio 
Grande, RS sob parecer $\mathrm{n}$ - $063-02 / 2012$, pelo Comitê de Ética em Pesquisa da Faculdade Anhanguera Educacional sob registro 108.745 e pela Plataforma Brasil sob o CAAE 05854912.1.0000.5372.

\section{RESULTADOS}

Participaram do estudo 21 gestantes, 6 no primeiro trimestre, 6 no segundo e 9 no terceiro trimestre gestacional. A média de idade foi de 25 anos. A grande maioria, 72\% relatou ser casada, $81 \%$ possuíam ensino fundamental completo e/ou incompleto como nível de escolaridade. Apenas 24\% trabalhavam e $86 \%$ dependiam da renda do pai da criança.

Tabela 1. Dados etários e antropométricos das gestantes usuárias de UBS, Rio Grande, RS, Brasil, 2012.

\begin{tabular}{|c|c|c|c|c|}
\hline Características & $1 \mathrm{TG}$ & $2 \mathrm{TG}$ & $3 \mathrm{TG}$ & Total \\
\hline Idade (anos) & $23,83 \pm 5,30$ & $23,83 \pm 6,51$ & $26,44 \pm 6,84$ & 24,95 \\
\hline $\begin{array}{l}\text { Massa corporal } \\
\text { Pré-gestação (kg) }\end{array}$ & $62,83 \pm 6,51$ & $74,67 \pm 14,40$ & $70,73 \pm 15,15$ & 69,43 \\
\hline $\begin{array}{l}\text { Massa corporal } \\
\text { gestacional (kg) }\end{array}$ & $64 \pm 7,97$ & $79,67 \pm 12,84$ & $79,67 \pm 13,68$ & 75,47 \\
\hline Estatura (m) & $1,58 \pm 0,46$ & $1,58 \pm 0,73$ & $1,61 \pm 0,62$ & 1,59 \\
\hline $\operatorname{IMC}^{3}\left(\mathrm{~kg} / \mathrm{m}^{2}\right)$ & $25,30 \pm 2,47$ & $30,52 \pm 5,36$ & $30,12 \pm 4,92$ & 28,86 \\
\hline
\end{tabular}

No gráfico 1 verifica-se que em $81 \%$ das gestantes declararam-se ativas relação ao nível habitual de atividade física fisicamente e as demais inativas fisicamente.

Grafico 1. Gestantes de acordo com a média dos resultados do IPAQ, Rio Grande, RS, Brasil, 2012.
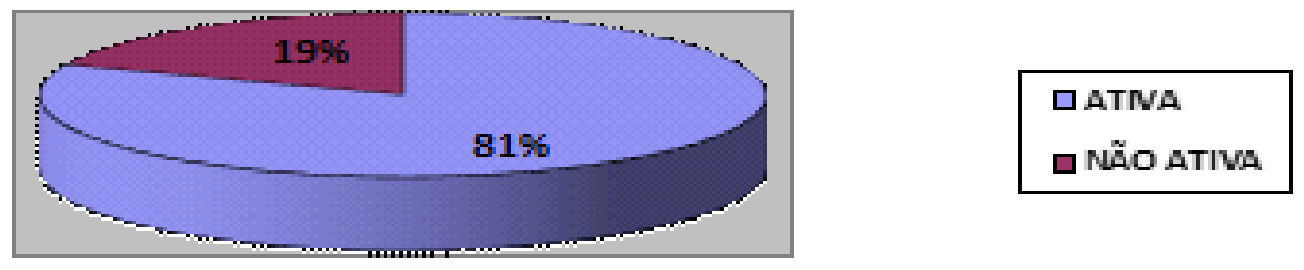

0 percentual referente ao quadro álgico das gestantes encontra-se no Gráfico 2. Percebe-se que $72 \%$ (15 das 21 gestantes) referiram presença de lombalgia, sendo que de todas as gestantes $19 \%$ (4) estão no $1^{\circ}$ TG,
Em relação a percepção de saúde, 81\% das gestantes relataram ter entre excelente e boa percepção. No que diz respeito aos hábitos de vida, $86 \%$ das gestantes não ingerem bebida alcoólica, $95 \%$ não são tabagistas e $100 \%$ relataram cuidar da alimentação.

Na tabela 1, estão apresentados os dados descritivos sobre as variáveis idade, massa corporal pré-gestacional, massa corporal gestacional, estatura e IMC relativos aos três grupos de gestantes avaliadas no estudo. 0 ganho de peso ponderal é verificado ao longo da gestação. 
Gráfico 2. Gestantes conforme resultados referentes ao questionário nórdico de sistemas osteomusculares, Rio Grande, RS, Brasil, 2012.

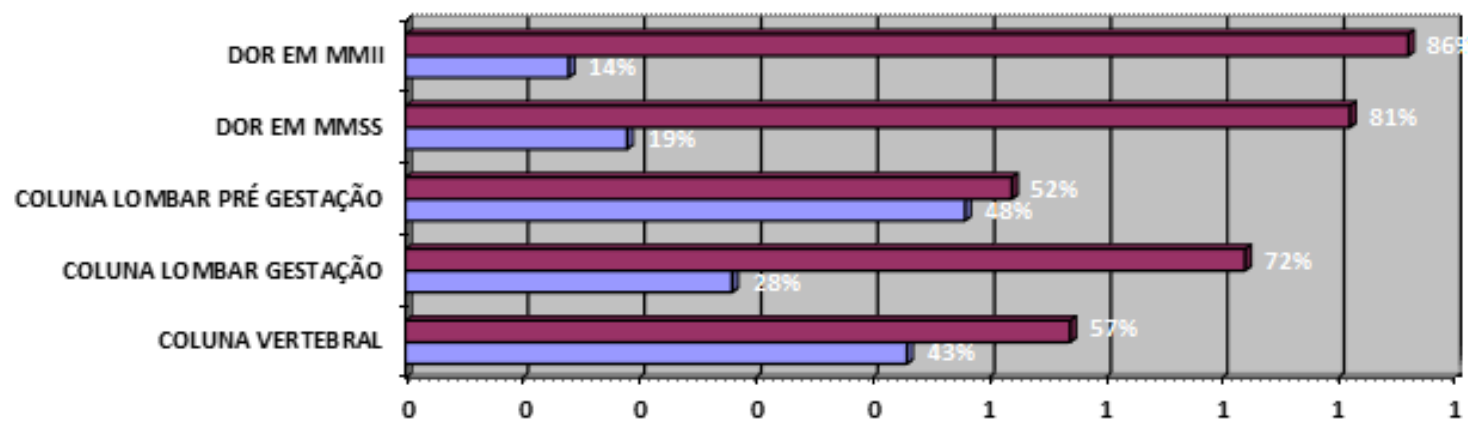

A tabela 2 apresenta média e desvio independente do trimestre gestacional padrão dos ângulos analisados nas gestantes, apresentaram um aumento da lordose referentes a lordose lombar de acordo com o lombar e 72\% relataram a presença de programa SAPo. Todas as gestantes, lombalgia gestacional.

Tabela 2. Gestantes segundo valores (média \pm desvio-padrão) dos ângulos analisados, Rio Grande, RS, Brasil, 2012.

\begin{tabular}{lccc}
\hline Ângulo & \multicolumn{3}{c}{ Média \pm Desvio padrão } \\
\hline LL & $1 \mathrm{TG}$ & $2 \mathrm{TG}$ & $3 \mathrm{TG}$ \\
& $14.28 \pm 3.39$ & $18.67 \pm 7.31$ & $14.97 \pm 4,79$ \\
\hline
\end{tabular}

\section{DISCUSSÃO}

A dor lombar é uma adversidade habitual na população, esse indício frequentemente acomete gestantes, principalmente quando o período gravídico está em fase avançado.

Nesse período, de modo geral, parte das gestantes costuma reclamar de algum tipo de desconforto, dentre eles a dor na região lombar. 0 presente estudo constatou que $72 \%$ das gestantes relataram sentir dor na coluna lombar, corroborando alguns trabalhos, que citam a predominância dessa dor entre 33\% e 77,6\%15-21. As condições maternas como: situação conjugal, ocupação, idade, escolaridade, duração da gestação, assim como o tipo de parto, interferem no estado de saúde da gestante e do bebêt ${ }^{18}$.

Estudo de revisão ${ }^{19}$, mostrou que independente da condição socioeconômica e dos hábitos de vida, a maioria das gestantes apresentavam sintomas e desconfortos musculoesqueléticos.

Corroborando com estes dados, pesquisa de base populacional ${ }^{20}$ demonstrou que a dor lombar pélvica atinge cerca de $50 \%$ de todas as gestações, principalmente a partir do $3^{\circ}$ trimestre gestacional.
Outra investigação ${ }^{19}$, que utilizou amostragem intencional não-probabilística com distribuição aleatória de casos, observou a ocorrência de lombalgia, em gestantes ativas e sedentárias e mulheres sedentárias. Notou-se prevalência de lombalgia (73\%), sendo mais frequente no terceiro trimestre gestacional em $48 \%$ destas $^{19}$. No estudo em questão, a prevalência de dor lombar apresentada foi de somente $1 \%$ a menos ao estudo supracitado, além de $29 \%$ no terceiro trimestre gravídico.

Outra pesquisa ${ }^{21}$ verificou maior prevalência de lombalgia a partir do $5^{\circ}$ mês de gestação $\left(2^{\circ} \mathrm{TG}\right)$. 0 quadro álgico na região lombar pode ocorrer em qualquer etapa do período gestacional, porém é marcante entre o $4^{\circ}$ ( $\left.2^{\circ} \mathrm{TG}\right)$ e o $7^{\circ}$ ( $\left(3^{\circ} \mathrm{TG}\right)$ mês.

No estado do Piauí foi realizado um estudo $^{22}$, do tipo não aleatório, em um hospital filantrópico, mostrando que a lordose lombar não foi um fator predisponente a lombalgia, indo de encontro ao presente estudo, pois conforme a tabela 2 , todos os trimestres gestacionais - TG tiveram aumento significativo no ângulo da lordose 
refletindo no agravamento do quadro álgico gestacional corroborando com o estudo.

\section{CONCLUSÃO}

No questionário QNSO, foi percebido alto índice de dor lombar nas gestantes sendo possível estabelecer uma associação das alterações posturais com a presença da dor lombar durante o período gestacional.

No presente estudo, foram verificadas alterações posturais na coluna lombar das gestantes nos três trimestres por meio da biofotogrametria que é um método de avaliação postural de alta complexidade, confiabilidade e importante no diagnóstico fisioterapêutico.

0 estudo realizado exibe certa limitação no que se refere ao tamanho da amostra, que ao se apresentar em número reduzido, permite considerar os resultados encontrados condizentes para a população em questão, contudo servindo de base sólida para outras pesquisas.

0 trabalho em questão espera despertar nos profissionais da saúde, o desejo de aprofundar novos estudos, idealizando uma pratica de exercícios físicos para as gestantes, independente da modalidade escolhida, contribuindo para um conhecimento mais amplo e adequado afim de contribuir com a qualidade de vida deste grupo, valorizando ainda mais a atuação do profissional como um agente importante na promoção da saúde da mulher.

\section{REFERÊNCIAS}

1. Almeida LGD, Constâncio JF, Santos CVS, Silva TH, Raposo MT. Análise comparativa das PE e PI máximas entre mulheres grávidas e não grávidas e entre grávidas de diferentes períodos gestacionais. Rev Saúde. 2005; 1(1):9-17.

2. Giacopini SM, Oliveira DV, Araújo APS. Benefícios e recomendações da prática de exercícios físicos na gestação. Rev Biosalus. 2016; 1(1):1-19.

3. Florindo JVC, Lima RDS. Tratamento fisioterapêutico em lombalgia gestacional: relato de caso. [Monografia]. Pindamonhangaba, SP: Faculdade de Pinhamonhangaba/FAPI; 2014. 37p.
4. Andrade MF. Existe correlação entre o ângulo de báscula pélvica, a força e a função dos músculos do assoalho pélvico?. [Dissertação]. Alfenas, MG: Universidade Federal de Alfenas/UFA; 2017. 69p.

5. Hobo TMW, Azevedo M. Lombalgia gestacional: prevalência, características e a interferência nas principais atividades da vida diária. Universitas: Ciênc Saúde. 2015; 13(2):71-8.

6. Moccellin AS, Driusso P. Avaliação do Controle Postural Dinâmico Durante a Gestação. Rev Bras Biomec. 2013; 14(27): 1.

7. Mann L, Kleinpaul JF, Teixeira CS, Konopka CK. Dor lombo-pélvica e exercício físico durante a gestação. Fisioter Mov. 2017; 21(2): 99-105.

8. Paula LF, Silva RGC. Correlação entre fatores associados à lombalgia, incapacidade lombar e as alterações nas curvaturas torácica e lombar no período gestacional. [Trabalho de Conclusão de Curso]. Matinhos, PR: Universidade Federal do Paraná/UFP; 2015.

9. Magazoni VS. Estudo correlacional entre a expansibilidade da caixa torácica e a capacidade vital pulmonar nos indivíduos portadores e não portadores de espondilite anquilosante. [Dissertação]. Uberlândia, MG: Centro Universitário do Triângulo/ UNIT; 2000.

10. Ricieri DV. Quantificação angular do movimento tóraco-abdominal, durante a ventilação tranqüila, através da Fotogrametria Computadorizada. [Dissertação]. Uberlândia, MG: Centro Universitário do Triângulo/ UNIT; 2000.

11. Baraúna MA, Morais EG, Oliveira ATM, Domingos LG, Sanchez HM, Silva RÂV, et al. Estudo correlacional e comparativo entre ângulo axilar e assimetria de ombro através de um protocolo biofotogramétrico. Fisioter Mov. 2017; 19(1):17-24.

12. Branco JC, Guido F, Jansen K, Giusti PH. Prevalência de sintomas osteomusculares em professores de escolas públicas e privadas do ensino fundamental. Fisioter Mov. 2017; 25(1):45-51.

13. Pinheiro FA, Tróccoli $\mathrm{T}$, Carvalho CV. Validação do Questionário Nórdico de Sintomas Osteomusculares como medida de 
morbidade. Rev Saúde Pública. 2002; 19. Ferreira CHJ, Nakano AMS. Reflexões 36(3):307-12.

sobre as bases conceituais que fundamentam

14. Craig CL, Marshall AL, Sjostrom M, Bauman AE, Booth ML, Ainsworth B, et al, International physical activity questionnaire: 12-country reliability and vality. Med Sci Sports Exerc. 2003; 35(8):1381-95.

15. Torquato E, Gerage A, Meurer S, Borges R, Silva M, Benedetti T. Comparação do nível de atividade física medido por acelerômetro e questionário IPAQ em idosos. Rev Bras Ativ Fís Saúde. 2016; 21(2):144-53.

16. Camargo MZ. Alinhamento postural da coluna no plano sagital de crianças eutróficas e acima do peso em fase pré-escolar e escolar: estudo longitudinal. [Tese]. Londrina, PR: Universidade Estadual de Londrina/UEL; 2018. 79p.

17. Ferreira EA, Duarte $M$, Maldonado EP, Burke TN, Marques AP. Postural assessment software (PAS/SAPO): validation and reliabiliy. Clinics. 2010; 65(7):675-81.

18. Barbosa EM, Oliveira ASS, Galiza DDF, Barros VL, Aguiar VF, Marques MB. Perfil sociodemográfico e obstétrico de parturientes de um hospital público. Rev a construção do conhecimento acerca da lombalgia na gestação. Rev Latinoam Enferm. 2001; 9(3):95-100.

20. Souza MS, Araújo CC, Castellen M, Sperandio FF. Afinal, toda gestante terá dor lombar? Femina. 2003; 31(3):273-7.

21. Santos MM, Gallo AP. Lombalgia gestacional: prevalência e características de um programa pré-natal. Arq Bras Ciênc Saúde. 2010; 1(3):4-6.

22. Toracio MAPC, Korelo RIG. Influência da articulação sacroilíaca na lombalgia gestacional e/ou dor pélvica posterior. Rev Fisioter Evid. 2010; 1(2):5-11.

CONTRIBUIÇÕES
Max dos Santos Afonso, Caroline
Henriques Graciano, Karine Fernandes
Ávila, Marla dos Santos Afonso,
Abelardo Soares de Oliveira Júnior
tiveram iguais contribuições e Fernanda
Burlani Neves fez revisão crítica e foi a
orientadora da pesquisa.
RENE. 2017; 18(2):227-33.

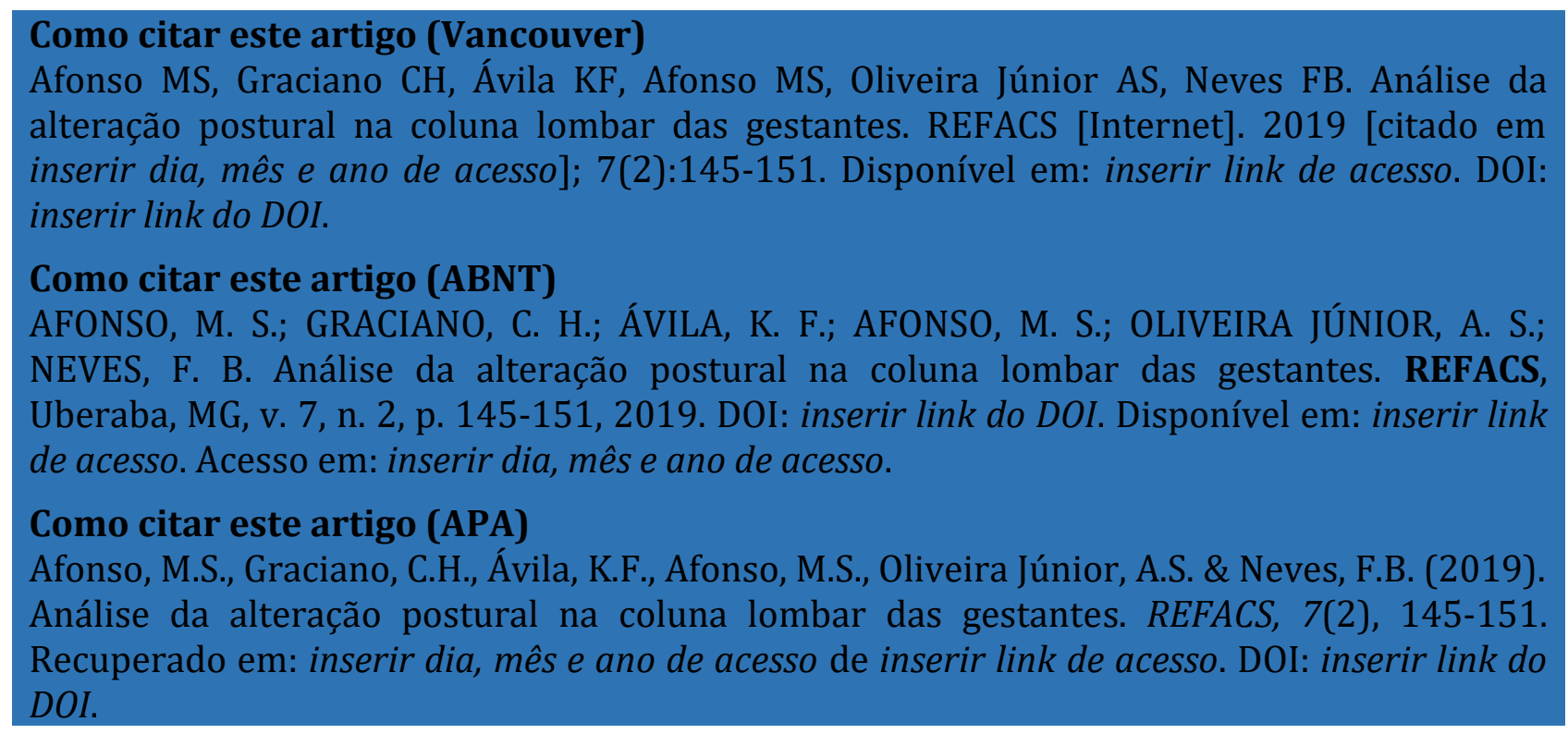

